\title{
Dermatologie
}

Kompass Dermatol 2022;10:52

Bitte beachten Sie: Aufgrund der Verbreitung des Coronavirus werden Veranstaltungen verschoben oder fallen kurzfristig aus. Bitte prüfen Sie vorher, ob ein Kongress stattfindet.

$\mid \begin{aligned} & \text { Dermatologie KOMPAKT \& } \\
& \text { PRAXISNAH } 2022 \\
& \text { 18.-20. Februar } 2022 \\
& \text { Wiesbaden, Deutschland }\end{aligned}$
\begin{tabular}{|l} 
Tagung DERM 2022 \\
11.-13. März 2022 \\
Verschoben auf 01.-03. Juli 2022 \\
Frankenthal, Deutschland
\end{tabular}
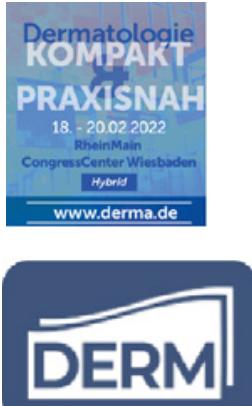

35th Nordic Congress of Dermatology and Venerology 19.-22. April 2022

Kopenhagen, Dänemark

FOBI 2022

12.-16. Juli 2022

München, Deutschland

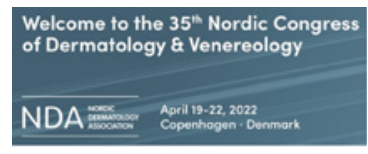

$\mathrm{FOBI}$ 2022

\section{FEBRUAR 2022}

RG Digital 2022 -

Dermatologisches Konsil in Berlin

23. Februar 2022

Virtuell

\section{ADF-Tagung}

23.-26. Februar 2022

Innsbruck, Österreich

\section{Deutscher Krebskongress}

23.-26. Februar 2022

Verschoben auf 13.-16. November 2022

Berlin, Deutschland

135. Tagung der Berliner

Dermatologischen Gesellschaft

26. Februar 2022

Virtuell

\section{MÄRZ 2022}

Essener Dermakonsil

05. März 2022

Virtuell

5. Lübecker Update: Entzündliche Hauterkrankungen

05. März 2022

Lübeck, Deutschland

34. Mainzer Allergie-Workshop

10.-11. März 2022

Virtuell

Neuigkeiten rund um die

Photodynamische Therapie

18.-19. März 2022

Virtuell

Global Vitiligo Foundation (GVF) Annual Scientific Symposium 2022 24. März 2022

Boston, USA
14. Akademie für Dermatoskopie, Auflicht- und Videomikroskopie Digital 25.-26. März 2022

Dresden, Deutschland

Xperience meets Enthusiams Die Vielfalt der Phlebologie praxisnah erleben

26. März 2022

Mönchengladbach, Deutschland

APRIL 2022

10. Bochumer Dermakonsil

02. April 2022

Bochum, Deutschland

\section{Potsdamer Dermakonsil}

02. April 2022

Potsdam, Deutschland

13. Nationale Versorgungskonferenz Psoriasis

08. April 2022

Virtuell

5. Düsseldorfer Forum für Kinderallergologie und Kinderdermatologie 09. April 2022

Düsseldorf, Deutschland

11. Berliner Lymphologisches Symposium

30. April 2022

Berlin, Deutschland

MAI 2022

10. Stuttgarter Dermakonsil

04. Mai 2022

Stuttgart, Deutschland

16. DEWU - Deutscher Wundkongress

04.-06. Mai 2022

Bremen, Deutschland
Lipödem Kongress 2022

06.-07. Mai 2022

Wien, Österreich

XXXVII. Jenaer Colloquium

allergologicum

07. Mai 2022

Jena, Deutschland

EADV-Symposium

12.-14. Mai 2022

Ljubljana, Slowenien

X. Marburger Allergie-Symposium 20.-21. Mai 2022

Marburg, Deutschland

14. Dermatologisches Alpenseminar "Allergie, Berufs- und Umweltdermatologie»

26.-28. Mai 2022

Grainau, Deutschland

JUNI 2022

14. Congress of the European Society of Contact Dermatitis 08.-10. Juni 2022

Amsterdam, Niederlande

Hautkrebs und Systemtherapie Symposium Rhein-Ruhr 11. Juni 2022

Bochum, Deutschland

JULI 2022

19th International Conference on Behcet's Disease 06.-08. Juli 2022 Athen, Griechenland 